\title{
Redox à la carte: Novel chemogenetic models of heart failure
}

\author{
Andrea Sorrentino ${ }^{1}$ and Thomas Michel $^{2}$ \\ ${ }^{1}$ Brigham and Women's Hospital Division of Cardiology \\ ${ }^{2}$ Brigham and Women's Hospital
}

May 5, 2020

\begin{abstract}
Many current animal models of heart failure are hampered by intrinsic methodological complexities, while other models yield only a subtle cardiac phenotype even after prolonged in vivo treatments. A new "chemogenetic" animal model of heart failure recapitulates a critical characteristic shared by many disease states that lead to heart failure in humans: an increase in redox stress in the heart. This "chemogenetic" approach exploits a recombinant yeast enzyme that can be dynamically and specifically activated in vivo to generate the reactive oxygen species (ROS) hydrogen peroxide (H2O2) in cardiac myocytes. Redox stress can be rapidly, selectively, and reversibly manipulated by chemogenetic generation of ROS in cardiac myocytes, yielding a new model of dilated cardiomyopathy. Treatment of animals with the angiotensin receptor blocker valsartan promotes recovery of ventricular function and resolution of adverse cardiac remodeling. This Mini-Review discusses in vivo chemogenetic approaches to manipulate and analyze oxidative stress in the heart.
\end{abstract}

\section{Defining a new animal model of heart failure}

Human heart failure is a multifactorial and heterogeneous clinical syndrome that can be caused by numerous etiologies that lead to diverse clinical manifestations in patients. Over the last several decades, animal models of human heart failure have served as informative experimental platforms to investigate the mechanisms, causes, and progression of disease, and have led to important clinical trials and novel therapies. From dogs to zebrafish to transgenic mice (and even yeast!), animal models have provided critical insights into the cellular and molecular derangements that lead to heart failure in humans. This mini-review focuses on rodent models of heart failure, which are widely used for drug screens and target validation ${ }^{15,22,27}$. Many of the current rodent models of heart failure are not amenable to high-throughput screening approaches, either because they are methodologically complex or have only a subtle cardiac phenotype. No single animal model of heart failure can fully replicate the complexities of human heart failure nor serve as the optimal experimental platform, yet with the burgeoning epidemic of heart failure $6,19,28,39$, new drugs and new models are needed. Recent advances in chemogenetics have led to the development of new animal models that may provide important insights into human heart failure pathophysiology and identify novel therapeutic targets.

\section{Historical perspective}

One of the most informative early animal models of heart failure was developed in rats in which the left coronary artery undergoes surgical ligation, leading to myocardial infarction, ventricular remodeling, and systolic failure. This animal model was exploited by Pfeffer et al. in 1979 to explore the correlation between cardiac infarct size and myocardial dysfunction ${ }^{24}$. These studies led to the development of novel therapies using angiotensin converting enzyme inhibitors to prevent adverse ventricular remodeling, and had a major impact on heart failure therapy and saved many lives ${ }^{22-25}$. Yet while this experimental MI model is an informative approach to investigate mechanisms of cardiac dysfunction and remodeling, a substantial fraction of the experimental animals may die from arrhythmia or cardiac rupture following coronary ligation, and 
the cardiac remodeling response in the surviving animals is variable and not consistent. Moreover, each animal requires an individual surgical intervention, which undermines even medium-throughput screening approaches. Another commonly used model of heart failure is the pressure-overload model, which can be used in either mice or rats. In this model, a ligature is tightened around the animal's aorta, which leads first to left ventricular hypertrophy, followed by ventricular dilation and cardiac dysfunction. This model does mimic aspects of the heart failure progression observed in human patients ${ }^{26}$, but even subtle differences in surgical techniques and between mouse strains can lead to different outcomes. With the growing incidence of cardiovascular diseases observed in patients with diabetes and obesity/metabolic syndrome, animal models of heart failure have been developed in which animals are exposed to specific diets (e.g. high-fat/high-sucrose feeding) in various models of type II diabetes mellitus. However, the cardiac dysfunction that is induced by these metabolic derangements may develop only after many months of treatment, and even then may often lead only to a subtle cardiac phenotype ${ }^{8}$. There are also many "knockout" mouse models in which individual genes that cause cardiomyopathy in humans have been targeted; these models have been quite informative, but the relevance of some of these monogenic cardiomyopathies to most forms of human heart failure is less clear ${ }^{2}$. A recent report ${ }^{31}$ described a "two hit" approach in mice involving both a dietary treatment (high fat feeding) along with a pharmacological intervention (nitric oxide synthase inhibition) that led to the development of a novel heart failure phenotype. Each of the animal models of heart failure has its strengths and weaknesses, and of course no model system can faithfully recapitulate the complex pathways and protean manifestations of heart failure in humans. There remains an urgent need for new and more tractable heart failure models that recapitulate key features of human heart failure.

Oxidative stress and oxidative eustress in the heart

Nearly all human cardiomyopathies are associated with high levels of reactive oxygen species (ROS), which can cause redox stress in cardiac tissues ${ }^{3,9}$. Elevated levels of ROS in cardiac tissues are also found in many heart failure models in animals ${ }^{1,9,15,29}$. By contrast, low levels of the stable ROS hydrogen peroxide $\left(\mathrm{H}_{2} \mathrm{O}_{2}\right)$ are critically involved in many physiological signal transduction cellular pathways in cardiac myocytes ${ }^{35}$ and in other tissues ${ }^{12,13,21,33}$. Indeed, ROS are the product of normal cellular metabolisma state termed oxidative eustress ${ }^{32}$ - and may derive from diverse sources, including mitochondrial electron transport or as the products of a broad range of intracellular enzymes. Pathological oxidative stress can derive from mitochondrial dysfunction, from the disruption of redox metabolism, and as a consequence of cellular inflammatory responses that lead to the activation of ROS-generating pathways. In many cardiovascular disease states, pathological oxidative stress is associated with disrupted cellular energetics, deleterious protein and DNA alterations, local inflammatory responses, and organ dysfunction ${ }^{3,}$ 16, 20, 29 .

A central problem in modeling oxidative stress derives from the eponymous reactivity of reactive oxygen species $^{7,14}, 38$. Opening up a bottle of hydrogen peroxide and pouring out a measured amount of the stuff over cultured cells does indeed elicit marked changes in cellular pathways- but does this experimental approach really recapitulate the complexities of intracellular oxidative stress? Certainly not! Conversely, adding high concentrations of "antioxidants" to cells can hardly replicate the convoluted intracellular enzymatic machinery that modulates redox metabolism. And even the detection of ROS is fraught: many of the assays and analytic approaches that are intended to detect ROS are plagued by lack of sensitivity and/or specificity ${ }^{14}$. These complex considerations undermine experimental approaches that seek to modulate and to measure oxidative stress in cells, and hamper efforts to prove that oxidative stress is actually causal for cardiovascular pathology, instead of merely being associated with redox derangements. In recent years, the field of redox biology has been transformed by the advent of new "chemogenetic" approaches to specifically generate ROS in cells, coupled with the development of novel biosensors to detect ROS . New insights into the roles of intracellular oxidants in cardiac function and dysfunction have emerged from recent studies that have combined chemogenetic approaches to generate ROS with the quantitation of ROS using highly specific biosensors.

A new chemogenetic heart failure model: "redox à la carte"

The newly-coined term "chemogenetics" ${ }^{37}$ refers to experimental systems in which the activity of novel 
recombinant proteins (receptors, enzymes, channels) in cells is dynamically regulated by the addition or removal of specific biochemicals (ligands, substrates), permitting the reversible modulation of cellular responses. Chemogenetic approaches have been used modulate intracellular redox state in mammalian cells by expressing a recombinant yeast D-amino acid oxidase (DAAO) construct that catalyzes the oxidation of D-amino acids to their corresponding $\alpha$-keto acids, in the process generating equimolar $\mathrm{H}_{2} \mathrm{O}_{2}$ (Figure 1$)^{11}$. Since mammalian tissues principally use L-amino acids ${ }^{11,18}$, the yeast D-amino acid oxidase is quiescent until D-amino acid substrate is provided. Modulation of intracellular redox balance can be reversibly manipulated by providing or withdrawing D-amino acids to cells or tissues expressing recombinant DAAO: in the presence of D-amino acids, DAAO generates $\mathrm{H}_{2} \mathrm{O}_{2}$ until such time as the D-amino acid substrate is removed. The exquisite selectivity with which intracellular oxidants can be manipulated using chemogenetics leads us to term this experimental approach "redox à la carte"- redox state can be dynamically and specifically modulated using a single very simple biochemical intervention. Moreover, after adding D-amino acids to cells expressing DAAO, the $\mathrm{H}_{2} \mathrm{O}_{2}$ that is generated by DAAO can be detected in cells that are co-transfected with the highly specific $\mathrm{H}_{2} \mathrm{O}_{2}$ biosensor $\mathrm{HyPer}^{4}$. This approach permits the concurrent chemogenetic generation of $\mathrm{H}_{2} \mathrm{O}_{2}$ (by DAAO) and detection of $\mathrm{H}_{2} \mathrm{O}_{2}$ by HyPer using live cell imaging 5, 34, 36 . These chemogenetic approaches in cultured cells have provided important insights into the intracellular metabolism and diffusion of $\mathrm{H}_{2} \mathrm{O}_{2}$ in different subcellular compartments.

Recently, these in vitro chemogenetic approaches have been extended to the in vivo setting: a new chemogenetic model of heart failure has been developed using DAAO to generate oxidative stress in cardiac myocytes in intact animals (rats or mice). There are many methodological challenges that needed to be addressed in order to make the transition from in vitro to in vivo chemogenetic approaches in the heart. In order to efficiently express DAAO in the heart, the yeast DAAO was targeted to cardiac myocytes by infecting mice or rats via tail vein injection using a recombinant DAAO virus that was constructed in the cardiotropic adeno-associated virus isotype 9 (AAV9) under control of the cardiac-specific cTnT promoter ${ }^{36}$ (Figure 1). The DAAO was expressed as a fusion protein along with the $\mathrm{H}_{2} \mathrm{O}_{2}$ biosensor HyPer in order to be able to simultaneously generate (DAAO) and detect (HyPer) $\mathrm{H}_{2} \mathrm{O}_{2}$. Robust DAAO-AAV9 expression was detected in cardiac myocytes within 3-4 weeks of virus infection- with only nominal protein expression in skeletal muscle, but in no other tissues ${ }^{36}$. Addition of the DAAO substrate D-alanine (but not L-alanine) to isolated cardiac myocytes led to rapid and robust $\mathrm{H}_{2} \mathrm{O}_{2}$ production, which was detected by the $\mathrm{H}_{2} \mathrm{O}_{2}$ biosensor HyPer that is expressed in the DAAO-HyPer fusion construct. These findings confirm that the recombinant DAAO-AAV9 construct is efficiently targeted and expressed in cardiac myocytes and verify the chemogenetic production of $\mathrm{H}_{2} \mathrm{O}_{2}$ in response to D-alanine in vitro. The stage was now set to study the in vivo effects of chronic oxidative stress in the heart by activating DAAO in living animals after infecting them with DAAO-AAV9.

So how does one selectively and effectively activate a stereoselective recombinant yeast enzyme once it has been delivered by a viral vector to the heart of a mammal? In vitro, the yeast DAAO is exquisitely stereoselective: L-alanine entirely fails to activate recombinant DAAO, while D-alanine markedly activates the enzyme ${ }^{36}$. From this observation, one might infer that amino acid transporters might also be strictly stereoselective- and this stereoselectivity would undermine the tractability of in vivoapproaches in which the D-amino acid substrate is provided to the DAAO-infected animal parenterally or enterally. Fortunately (or fortuitously), most of the mammalian metabolite transporters responsible for the uptake of amino acids (encoded by the SLC solute carrier gene family) are not very stereoselective ${ }^{30}$. When mice or rats infected with DAAO-AAV9 are provided with drinking water supplemented with D-alanine, the animals drink the water avidly, and soon develop a dilated cardiomyopathy. Within 3 weeks of D-alanine feeding, the animals developed a striking reduction in ejection fraction and global longitudinal strain, accompanied by a significant enlargement of the left ventricular chamber and increased heart weight ${ }^{36}$ (Figure 2). As has been found in human heart failure, there was a marked increase in the abundance of transcripts encoding atrial natriuretic peptide (ANP), brain natriuretic peptide (BNP), and cardiac beta myosin heavy chain ( $\beta$-MHC), accompanied by a decrease in cardiac alpha-myosin heavy chain $\left(\alpha\right.$-MHC) transcript levels ${ }^{10,17,34,36}$. Moreover, markers of RNA oxidation were increased. Despite all these markers of cardiac dysfunction, longer-term 
exposure to D-alanine did not yield a further decline in ventricular function. It seems plausible that chronic exposure to oxidative stress elicits a broad range of protective compensatory responses in the heart, reflected by an increase in transcripts encoding "antioxidant" enzymes and by an increase in intracellular glutathione levels ${ }^{36}$. Despite marked ventricular dysfunction, there was no evidence of interstitial cardiac fibrosis even when the dilated cardiomyopathy phenotype was at its most extreme ${ }^{36}$, suggesting that the cardiac dysfunction in this model is independent from fibrotic processes.

In order for chemogenetic heart failure to be a useful model for drug screening and target validation, the phenotype needs to be reversible. The striking dilated cardiomyopathy seen in animals infected with DAAOAAV9 and fed D-alanine was found to fully resolve when the animals were treated with the angiotensin receptor blocker valsartan- administered either alone or with the neprilysin inhibitor sacubitril (Figure 2). This salutary response to drug treatment was seen even in the face of ongoing oxidative stress. Longerterm exposure to oxidative stress did eventually lead to the development of cardiac fibrosis, which was seen even when the heart failure phenotype had fully resolved with drug treatment. These observations lead to the important conclusion:significant cardiac dysfunction and ventricular remodeling may develop in the absence of cardiac fibrosis, while interstitial cardiac fibrosis may persist even after contractile dysfunction and adverse remodeling have resolved. These findings suggest that cardiac fibrosis cannot necessarily be used as a surrogate marker for cardiac dysfunction, nor does cardiac dysfunction necessarily involve cardiac fibrosis.

\section{Advantages and disadvantages of the chemogenetic model of heart failure}

The chemogenetic approach used to generate $\mathrm{H}_{2} \mathrm{O}_{2}$ in the heart has resulted in a robust and easily manipulated model for cardiac dysfunction that may lead to development of new therapeutic targets to treat or prevent heart failure. The chemogenetic model of heart failure recapitulates many commonly observed features of human heart failure and allows us to distinguish cardiac myocyte-specific pathology from the pleiotropic changes characteristic of other "interventional" animal models of heart failure. While these observations help to prove causality, this does not establish that oxidative stress is the only cause and isnecessary for development of heart failure. Indeed, the broad spectrum of heart failure syndromes suggests that oxidative stress may play a central role in some forms of heart failure but be less important in others. We acknowledge that intracellular generation of cytosolic $\mathrm{H}_{2} \mathrm{O}_{2}$ by a recombinant yeast enzyme in cardiac myocytes does not necessarily recapitulate all the complex alterations in cardiac and systemic redox metabolism that are associated with cardiomyopathies in human patients. Despite these limitations, chemogenetic approaches have been used to devise an informative and tractable new animal model of heart

failure caused by oxidative stress. Further study of his robust and reversible animal model may lead to the identification of novel therapeutic targets for the prevention and treatment of heart failure.

Words: 2,220

\section{References}

[1] Battiprolu, P. K., Hojayev, B., Jiang, N., Wang, Z. V., Luo, X., Iglewski, M., Shelton, J. M., Gerard, R. D., Rothermel, B. A., Gillette, T. G., Lavandero, S., and Hill, J. A. (2012) Metabolic stress-induced activation of FoxO1 triggers diabetic cardiomyopathy in mice, J Clin Invest 122 , 1109-1118.

[2] Becher, P. M., Jugdutt, B. I., Baugh, J., and Schmack, B. (2016) Experimental Heart Failure Models and Their Pathophysiological Characterization, Biomed Res Int 2016 , 2538263-2538263.

[3] Bertero, E., and Maack, C. (2018) Metabolic remodelling in heart failure, Nat Rev Cardiol 15 , 457-470.

[4] Bilan, D. S., and Belousov, V. V. (2016) HyPer Family Probes: State of the Art, Antioxid Redox Signal $24,731-751$.

[5] Bogdanova, Y. A., Schultz, C., and Belousov, V. V. (2017) Local Generation and Imaging of Hydrogen Peroxide in Living Cells, Curr Protoc Chem Biol 9 , 117-127.

[6] Braunwald, E. (2013) Heart failure, JACC Heart Fail1 , 1-20. 
[7] Brown, D. I., and Griendling, K. K. (2015) Regulation of signal transduction by reactive oxygen species in the cardiovascular system, Circ Res $116,531-549$.

[8] Bugger, H., and Abel, E. D. (2009) Rodent models of diabetic cardiomyopathy, Disease Models Éamp; Mechanisms 2, 454-466.

[9] Burgoyne, J. R., Mongue-Din, H., Eaton, P., and Shah, A. M. (2012) Redox signaling in cardiac physiology and pathology, Circ Res 111, 1091-1106.

[10] Carniel, E., Taylor, M. R., Sinagra, G., Di Lenarda, A., Ku, L., Fain, P. R., Boucek, M. M., Cavanaugh, J., Miocic, S., Slavov, D., Graw, S. L., Feiger, J., Zhu, X. Z., Dao, D., Ferguson, D. A., Bristow, M. R., and Mestroni, L. (2005) Alpha-myosin heavy chain: a sarcomeric gene associated with dilated and hypertrophic phenotypes of cardiomyopathy, Circulation 112 , 54-59.

[11] D'Aniello, A., D'Onofrio, G., Pischetola, M., D'Aniello, G., Vetere, A., Petrucelli, L., and Fisher, G. H. (1993) Biological role of D-amino acid oxidase and D-aspartate oxidase. Effects of D-amino acids, J Biol Chem 268 , 26941-26949.

[12] Finkel, T. (2003) Oxidant signals and oxidative stress, Curr Opin Cell Biol 15 , 247-254.

[13] Finkel, T. (2011) Signal transduction by reactive oxygen species, J Cell Biol 194 , 7-15.

[14] Griendling, K. K., Touyz, R. M., Zweier, J. L., Dikalov, S., Chilian, W., Chen, Y.-R., Harrison, D. G., Bhatnagar, A., and American Heart Association Council on Basic Cardiovascular, S. (2016) Measurement of Reactive Oxygen Species, Reactive Nitrogen Species, and Redox-Dependent Signaling in the Cardiovascular System: A Scientific Statement From the American Heart Association, Circulation research 119, e39-e75.

[15] Houser, S. R., Margulies, K. B., Murphy, A. M., Spinale, F. G., Francis, G. S., Prabhu, S. D., Rockman, H. A., Kass, D. A., Molkentin, J. D., Sussman, M. A., and Koch, W. J. (2012) Animal models of heart failure: a scientific statement from the American Heart Association, Circ Res 111, 131-150.

[16] Kanaan, G. N., and Harper, M. E. (2017) Cellular redox dysfunction in the development of cardiovascular diseases, Biochim Biophys Acta Gen Subj 1861, 2822-2829.

[17] Kerkela, R., Ulvila, J., and Magga, J. (2015) Natriuretic Peptides in the Regulation of Cardiovascular Physiology and Metabolic Events, J Am Heart Assoc 4, e002423.

[18] Matlashov, M. E., Belousov, V. V., and Enikolopov, G. (2014) How much $\mathrm{H}(2) \mathrm{O}(2)$ is produced by recombinant D-amino acid oxidase in mammalian cells?, Antioxidants $\& 6$ redox signaling 20 , 1039-1044.

[19] McMurray, J. J. (2010) Clinical practice. Systolic heart failure, N Engl J Med 362 , 228-238.

[20] Murphy, M. P., Holmgren, A., Larsson, N. G., Halliwell, B., Chang, C. J., Kalyanaraman, B., Rhee, S. G., Thornalley, P. J., Partridge, L., Gems, D., Nystrom, T., Belousov, V., Schumacker, P. T., and Winterbourn, C. C. (2011) Unraveling the biological roles of reactive oxygen species, Cell Metab 13, 361-366.

[21] Ogura, S., and Shimosawa, T. (2014) Oxidative stress and organ damages, Curr Hypertens Rep 16 , 452 .

[22] Patten, R. D., and Hall-Porter, M. R. (2009) Small animal models of heart failure: development of novel therapies, past and present, Circulation. Heart failure 2 , 138-144.

[23] Pfeffer, J. M., Pfeffer, M. A., and Braunwald, E. (1985) Influence of chronic captopril therapy on the infarcted left ventricle of the rat, Circulation research 57, 84-95.

[24] Pfeffer, M. A., Pfeffer, J. M., Fishbein, M. C., Fletcher, P. J., Spadaro, J., Kloner, R. A., and Braunwald, E. (1979) Myocardial infarct size and ventricular function in rats, Circulation research $44,503-512$.

[25] Pfeffer, M. A., Pfeffer, J. M., Steinberg, C., and Finn, P. (1985) Survival after an experimental myocardial infarction: beneficial effects of long-term therapy with captopril, Circulation72 , 406-412. 
[26] Richards, D. A., Aronovitz, M. J., Calamaras, T. D., Tam, K., Martin, G. L., Liu, P., Bowditch, H. K., Zhang, P., Huggins, G. S., and Blanton, R. M. (2019) Distinct Phenotypes Induced by Three Degrees of Transverse Aortic Constriction in Mice, Sci Rep 9 , 5844.

[27] Riehle, C., and Bauersachs, J. (2019) Small animal models of heart failure, Cardiovascular Research 115 , 1838-1849.

[28] Roger, V. L. (2013) Epidemiology of Heart Failure, Circulation Research 113 , 646-659.

[29] Roul, D., and Recchia, F. A. (2015) Metabolic alterations induce oxidative stress in diabetic and failing hearts: different pathways, same outcome, Antioxidants 86 redox signaling22 , 1502-1514.

[30] Sahoo, S., Aurich, M. K., Jonsson, J. J., and Thiele, I. (2014) Membrane transporters in a human genome-scale metabolic knowledgebase and their implications for disease, Frontiers in Physiology5 , 91.

[31] Schiattarella, G. G., Altamirano, F., Tong, D., French, K. M., Villalobos, E., Kim, S. Y., Luo, X., Jiang, N., May, H. I., Wang, Z. V., Hill, T. M., Mammen, P. P. A., Huang, J., Lee, D. I., Hahn, V. S., Sharma, K., Kass, D. A., Lavandero, S., Gillette, T. G., and Hill, J. A. (2019) Nitrosative stress drives heart failure with preserved ejection fraction, Nature $568,351-356$.

[32] Sies, H. (2017) Hydrogen peroxide as a central redox signaling molecule in physiological oxidative stress: Oxidative eustress, Redox Biol 11, 613-619.

[33] Sies, H. (2015) Oxidative stress: a concept in redox biology and medicine, Redox biology 4, 180-183.

[34] Sorrentino, A., Steinhorn, B., Troncone, L., Saravi, S. S. S., Badole, S., Eroglu, E., Kijewski, M. F., Divakaran, S., Di Carli, M., and Michel, T. (2019) Reversal of heart failure in a chemogenetic model of persistent cardiac redox stress, Am J Physiol Heart Circ Physiol 317, H617-h626.

[35] Steinhorn, B., Sartoretto, J. L., Sorrentino, A., Romero, N., Kalwa, H., Abel, E. D., and Michel, T. (2017) Insulin-dependent metabolic and inotropic responses in the heart are modulated by hydrogen peroxide from NADPH-oxidase isoforms NOX2 and NOX4, Free Radic Biol Med 113 , 16-25.

[36] Steinhorn, B., Sorrentino, A., Badole, S., Bogdanova, Y., Belousov, V., and Michel, T. (2018) Chemogenetic generation of hydrogen peroxide in the heart induces severe cardiac dysfunction, Nat Commun 9 , 4044 .

[37] Urban, D. J., and Roth, B. L. (2015) DREADDs (designer receptors exclusively activated by designer drugs): chemogenetic tools with therapeutic utility, Annu Rev Pharmacol Toxicol 55 , 399-417.

[38] Zhang, Y., Tocchetti, C. G., Krieg, T., and Moens, A. L. (2012) Oxidative and nitrosative stress in the maintenance of myocardial function, Free Radic Biol Med 53 , 1531-1540.

[39] Ziaeian, B., and Fonarow, G. C. (2016) Epidemiology and aetiology of heart failure, Nat Rev Cardiol 13 , 368-378.

FIGURES

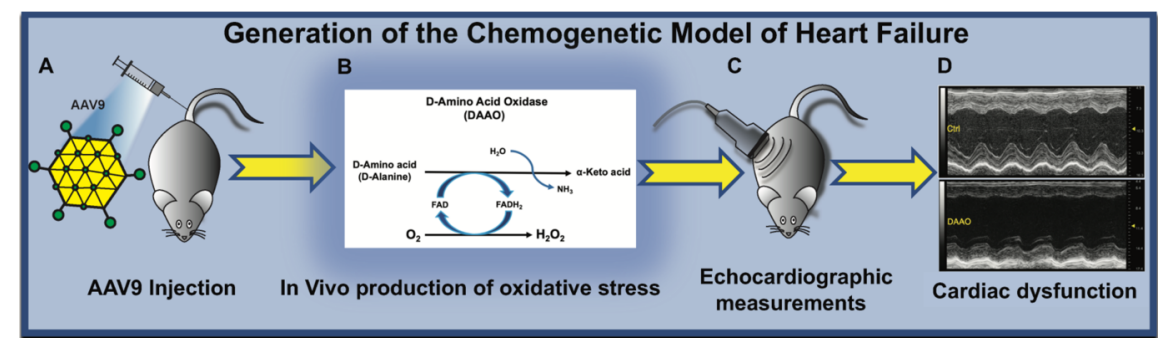

Figure 1. Generating a chemogenetic model of heart failure caused by oxidative stress. A) A 
recombinant cardiotropic adeno-associated virus serotype 9 (AAV9) expressing a yeast D-amino acid oxidase (DAAO) under control of the cardiac-specific cTnT promoter was constructed and injected via tail vein into rats or mice. B) 4 weeks following infection with either control AAV9 or DAAO-AAV9, the DAAO substrate D-alanine was provided in the animals' drinking water. D-alanine feeding leads to the activation of DAAO, which catalyzes the oxidation of D-alanine to its corresponding $\alpha$-keto acid (pyruvate), along with equimolar generation of $\mathrm{H}_{2} \mathrm{O}_{2}$ and ammonia $\mathrm{C}$ ) The effects of in vivo generation of oxidative stress in cardiac myocytes were monitored weekly by echocardiography. D) After 4 weeks of chemogenetic oxidative stress, the DAAO-infected animals develop severe cardiac dysfunction, with ventricular dilation and decreased ejection fraction. Shown are representative echocardiographic images of the left ventricle of control AAV9 (above) or DAAO-AAV9-infected rats (below).

Modified from Steinhorn B, Sorrentino A, Badole S, Bogdanova Y, Belousov, V., and Michel, T. (2018) Chemogenetic generation of hydrogen peroxide in the heart induces severe cardiac dysfunction, Nature Commun 9, 4044 .

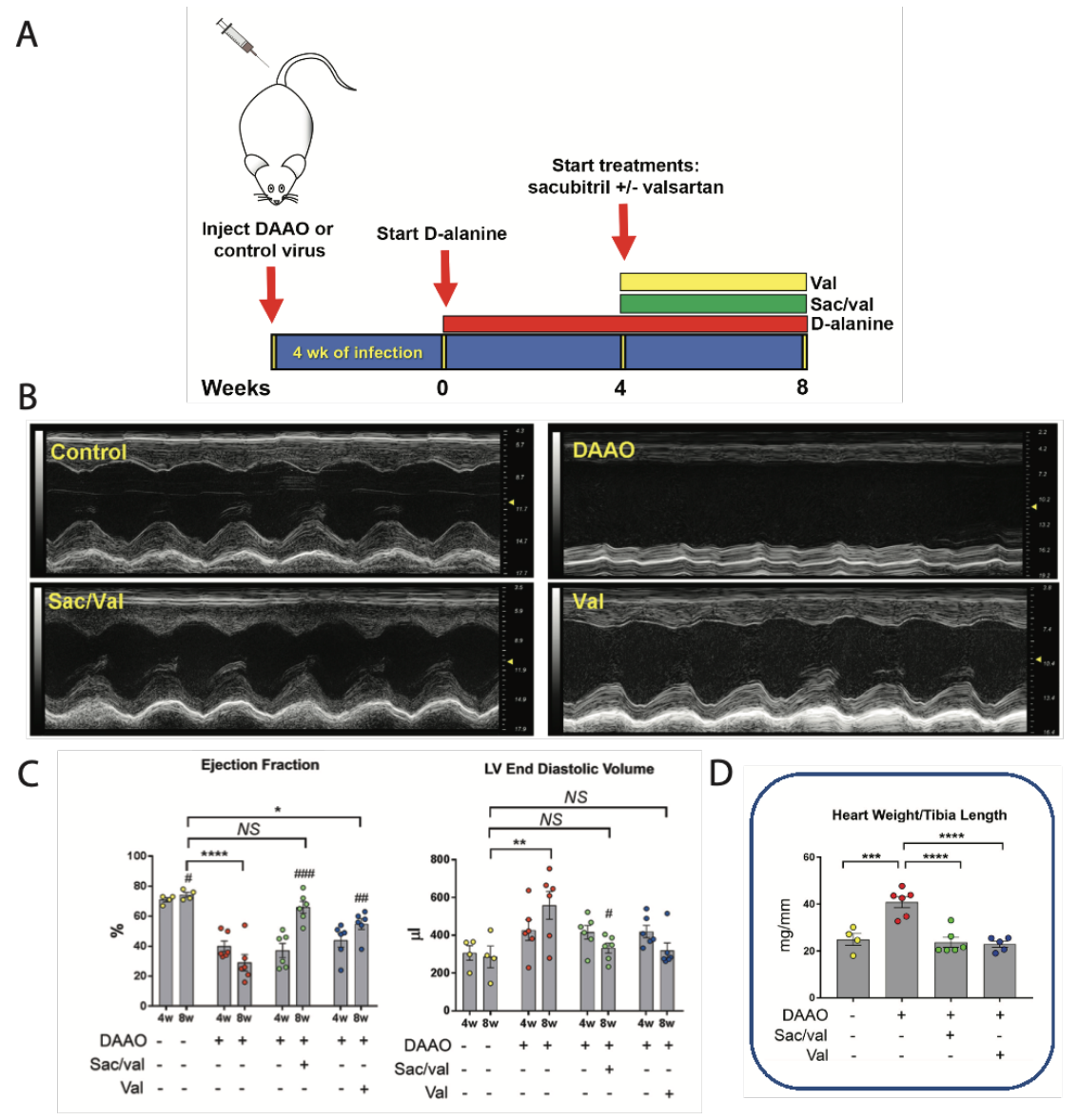

Figure 2. Reversal of the chemogenetic model of heart failure following drug treatments. A, Schematic showing timeline for induction of chemogenetic heart failure by AAV9-DAAO infection plus Dalanine feeding, followed by treatment of rats with the heart failure drugs sacubitril/valsartan or valsartan. B, Representative echocardiographic measurements in control or DAAO-infected rats, followed by treatments with sacubitril/valsartan or valsartan alone. C-D, Quantitative analyses of pooled data showing ejection fraction, left ventricular end diastolic volume and measurements of heart weight normalized to tibia length in DAAO-AAV9-infected animals following treatments as shown. 
Modified from Sorrentino, A. et al. Reversal of heart failure in a chemogenetic model of persistent cardiac redox stress. Am J Physiol Heart Circ Physiol 317, H617-h626 (2019). 